\title{
Developing of Intelligence Walking Stick and Mobile Application for Elderly Health Care Using the Internet of Things
}

\author{
https://doi.org/10.3991/ijim.v14i14.14813 \\ Sumitra Nuanmeesri $(\bowtie)$ \\ Suan Sunandha Rajabhat University, Bangkok, Thailand \\ sumitra.nuessru.ac.th \\ Lap Poomhiran \\ King Mongkut's University of Technology North Bangkok, Bangkok, Thailand
}

\begin{abstract}
Nowadays, Thailand is stepping into an aging society. This research purposes developing the intelligence walking stick for the elderly in terms of the health care system by applied the internet of things devices and biometric sensors in a real-time system. The heart rate, blood pressure, oxygen saturation, and temperature were measured at the finger of the elderly that holding the intelligence walking stick. All data can monitor and display on mobile devices. The intelligence walking stick system was evaluated by twenty users who are five experts and fifteen elderly in Ratchaburi province. As a result of the mean value at 4.15 and 4.61 by experts and elderly, respectively. Moreover, the acceptance of the intelligence walking stick for the elderly that could be detected by elderly people while being used has high consensus. This study indicates that the developed intelligence walking stick by using IoT can help and improve the daily living of the elderly at high level.
\end{abstract}

Keywords—Elderly, health care, intelligence walking stick, internet of things.

\section{Introduction}

Nowadays, Thailand is stepping into an aging society. Currently, there are $16,396,128$ Thai people aged sixty years and over, which are of Thai nationality was called elderly person [1], with 6,228,766 female seniors elderly and 5,083,681 male seniors elderly, accounting for the proportion of females and males can get $55 \%$ and $44 \%$, respectively [2]. These older people, therefore, need to take care of themselves. Especially in the case of wanting to go outside to do activities such as walking, exercising in the park, traveling to buy or buy certain products, traveling for leisure travel, they need someone or something to help them while doing any activities explained above.

For this digital age, most people have to work outside the home. They were resulting in not having much time to look after or be close to the elderly family members. Thus, these older people must be able to be self-reliant to be able to carry on their 
activities or activities without burden to their family members. However, people in the family of these elderly persons are still concerned about the welfare of the elderly while leaving home alone. Because of many older people who are sick or have certain medical conditions, such as heart disease, high blood pressure, are causing more anxiety due to not staying close to the elderly all the time or they walking out of their home.

In the market today, there are many types of measurement tools for health care for the elderly such as heart rate monitor, blood pressure monitors such as the Omron blood pressure monitor that has the cuff for pressure measurement [3]. Nevertheless, these tools are unique devices, and they are not easily portable. In the case of small devices, the price will be quite high as well. For example, the Samsung Galaxy S9 mobile phone, which has a heart rate sensor and blood pressure monitor as well [4], or the HeartGuide is a revolutionary bracelet watch with a blood pressure monitor and pulse monitor [5]. Which these tools may not be necessary or are everyday items that these elderly people pick up or use regularly.

The walking sticks are another tool that helps the elderly perform various activities, especially the ability to help walk for the elderly. It is a device that is sometimes like a part of the body of the elderly that may not be missing from daily life. In most cases, these canes or walking sticks manufactured to support while standing or walking. However, there are still other capabilities added to the walking stick with the new technology, for example, it including a flashlight, radio receiver, and music player. In some cases, it has abilities for calling and answer the incoming call on itself [6]. Considering the care of the elderly, there is not yet a walking stick that has been developed to help or promote health care for the elderly. In the market, it has only the features of asking for help with sound and light alarms on the walking stick.

With the internet and communication technology changing rapidly, resulting in a new device that can be widely used to create benefits and help improve life and wellbeing. The internet of things (IoT) is a new technology that brings a huge of electronic devices that allow connect and communicate on the internet. For example, the auto robot detects and collects the plastic floating garbage then sent a short message to social media via IoT devices . Another example, the automatic system in fish farming, it helps the entrepreneur to maintain the water quality in the fish tank that can monitoring in real-time on the internet. In a field of the internet of things, there are many small bio-sensors for detecting and measuring in terms of health care, such as heart rate sensors, blood pressure sensors, body temperature sensors.

Therefore, this research aims to develop intelligent walking sticks to help care for the health of the elderly and used as a tool to track the elderly in real-time through the application on the smartphone. This paper has consisted of different sections, as follows. Section 2 describes the principles and concepts of the design and development of the intelligence walking stick and the mobile application. In Section 3, there are the results of the effectiveness evaluation of the mobile application. Finally, a conclusion is provided in Section 4. 


\section{Methodology}

The research methods for studying the development of the intelligence walking stick for the elderly include the following steps:

\subsection{The intelligence walking stick design}

In this phase, the intelligence walking stick designed for the elderly in terms of health care. The researchers gathering information related to the biosensors and IoT devices that can be tracking the elderly persons when walking out of their home. Thus, in the research, there are fourteen components for the circuit design in the IoT system. The IoT devices and sensors illustrated in Figure 1.

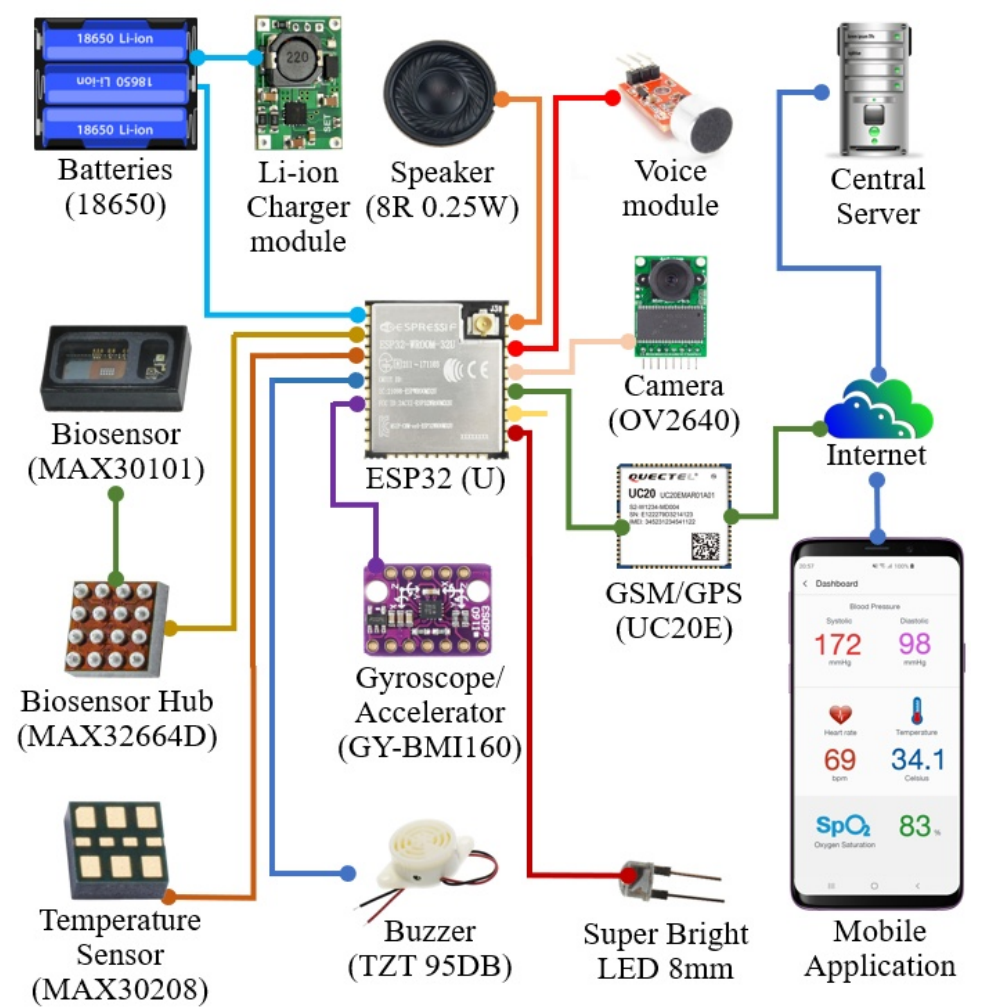

Fig. 1. Primary sensors and IoT devices of the intelligence walking stick for elderly health care

All details of each sensor and electronic device are explained as follows.

1. ESP32 module: The ESP32 is the microcontroller, which is the leading electronic control unit (ECU) in this work. In general, there are several modules for the 
ESP32 board. The ESP32-WROOM-32U is the smallest in the ESP32 series. Because the handle of a walking stick has a few spaces to contain any devices and circuit. So, any smallest device is suitable in this situation. The dimensions of the ESP32-WROOM-32U are $19.2 \times 18 \times 3.1$ millimeters.

2. Temperature sensor: The generic temperature sensor cannot measure the body temperature directly. In this research, the MAX30208 sensor was applied for measuring the body temperature. This sensor is a digital temperature sensor that is developed by Maxim Integrated company. It has 16-bit resolution and communicates via the I2C interface with dimensions at $2 \times 2 \times 0.75$ millimeters.

3. Biosensor or Biometric sensor: In this research, the MAX30101 is used to measure the biological data. It consists of a heart rate monitoring, pulse oximeter or blood oxygen saturation monitoring, and blood pressure trending monitoring. This sensor can measure the biological data for both finger and wrist. It is great to be able to combine all three of these measurements into a single sensor. Furthermore, it is advantageous when installing under the restricted area of the intelligence walking stick. The MAX30101 has tiny dimensions at 5.6 × 3.3 x 1.55 millimeters with 14pins on the optical module.

4. Biosensor hub or Biometric sensor hub: The MAX32664D is conducted for controlling and measuring the biological data. This microchip includes the algorithm for computing the biological data sending from the biosensor. Notably, the MAX30101 requires this biometric hub for measuring the blood pressure, heartbeat, and pulse blood oxygen saturation. According to the MAX32664D datasheet, the MAX30101 can integrate into MAX32644D as Figure 2 [7].

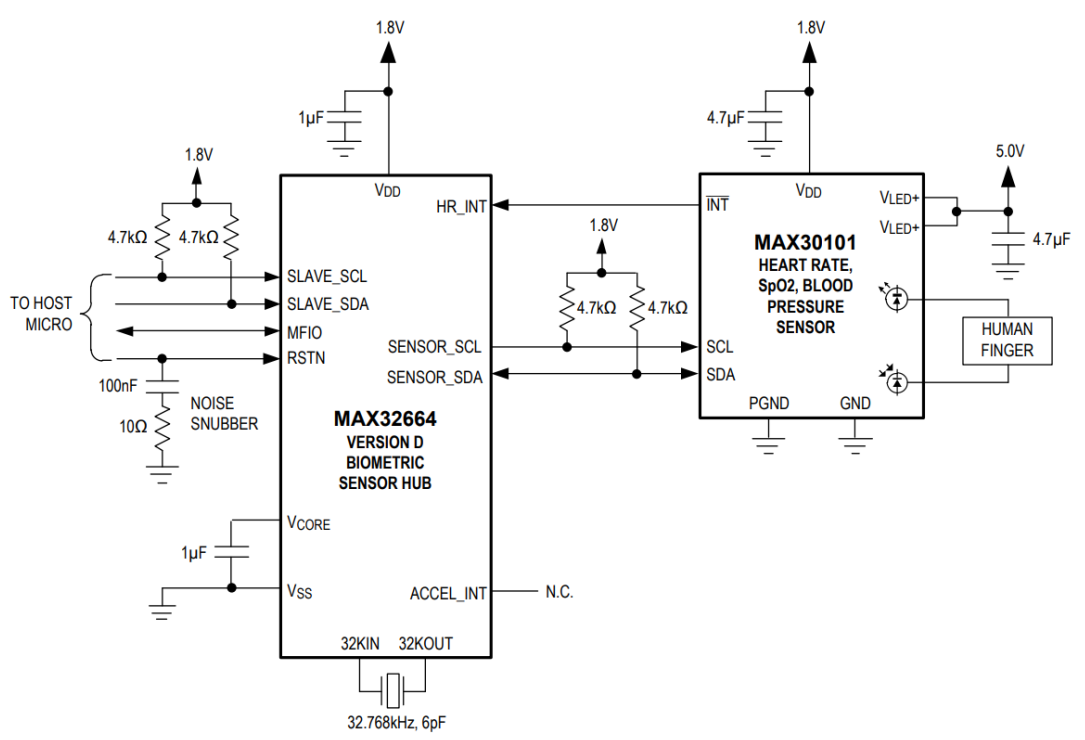

Fig. 2. Circuit integrated between MAX30101 and MAX32664 
5. GSM/GPS module: The Quectel UC20E was conducted to develop an intelligence walking stick. It supports a wide frequency range from 850 to 2100 megahertz. This module has benefits in calling and answering the phone or send a short message. Moreover, it can connect to the internet with 14.4 megabits per second for a maximum of downloading, and 5.76 megabits per second for downloading. Furthermore, GPS tracking is included to receive the data from the GNSS satellites, and then the current location was integrated and displayed on Google Maps.

6. Camera module: The camera module is applied for snapping an image and streaming the video from the intelligence walking stick. In this research, the OV2640 camera module selected because it comes with high resolution up to two megapixels.

7. Gyroscope and gravity acceleration sensor: This sensor is chosen to detect the status or position of the elderly who is holding the intelligence walking stick while standing or walking. If the elderly fall over with it, this situation can be detected by this sensor. The GY-BMI160 is selected in this research. It has six-axis in total, three-axis for accelerator meter, and three-axis for gyroscope meter.

8. Voice and speaker module: Voice module is conducted as a microphone of sound input for calling and answering the phone. The speaker is used to listen to the voice that communicates via the GSM module. In general, the speaker should have the resistor $8 \mathrm{ohms}$ and power-driven at least 0.25 watt.

9. Buzzer: The buzzer TZT 95DB is acted as sound alarming to the nearby people. This buzzer comes with high-decibel.

10. The light-emitting diode: The light-emitting diode or LED is designed for light alarming.

11. Battery and rechargeable module: The intelligence walking stick is rechargeable via the Li-ion charger module, which used to recharge the energy to the 18650 batteries.

\subsection{The design of the mobile application}

The information was collected from the data of the intelligence walking stick for developing the features in the mobile application. The consistency of the contents was analyzed by five experts in the field of information technology and elderly health care. Accordingly, the information was taken from the experts to determine the IOC value by using the formula as presented in the equation [8]:

$$
\mathrm{IOC}=\frac{\sum \mathrm{R}}{\mathrm{N}}
$$

$\sum \mathrm{R}$ is the sum of the scores evaluated by experts.

$\mathrm{R}$ is the score evaluated by experts.

$\mathrm{N}$ is the number of experts.

The features in the mobile application were assessed by five experts specializing in the field of information technology and elderly health. The Index of item objective congruence (IOC) was applied to the evaluation [9][10][11][12] to determine scores. If the criteria measured by the IOC value of each indicator was higher than 0.5 (the 
highest IOC value is 1), it could be implied that the features met the objectives and possessed the contents suitable for the operation, showing that the features worked effectively. For instance, if the IOC value of each indicator was 0.8 , it means the features met the objective and were suitable for use in the evaluation. The experts were able to give a rating by considering the assessment criteria, as shown in Table 1.

Table 1. The criteria of content consistency for identifying features in the mobile application development

\begin{tabular}{|c|l|}
\hline Rating criteria & \multicolumn{1}{|c|}{ Meaning } \\
\hline 1 & $\begin{array}{l}\text { There is content consistency, showing the suitability to be used as a feature in the applica- } \\
\text { tion. }\end{array}$ \\
\hline 0 & $\begin{array}{l}\text { There is uncertainty in the content consistency, resulting in its inappropriateness to be } \\
\text { used as a feature in the application }\end{array}$ \\
\hline-1 & $\begin{array}{l}\text { There is neither content consistency nor appropriateness to be used as a feature in the } \\
\text { application. }\end{array}$ \\
\hline
\end{tabular}

Figure 3 summarizes the IOC evaluation to identify features for the operation of the mobile application.

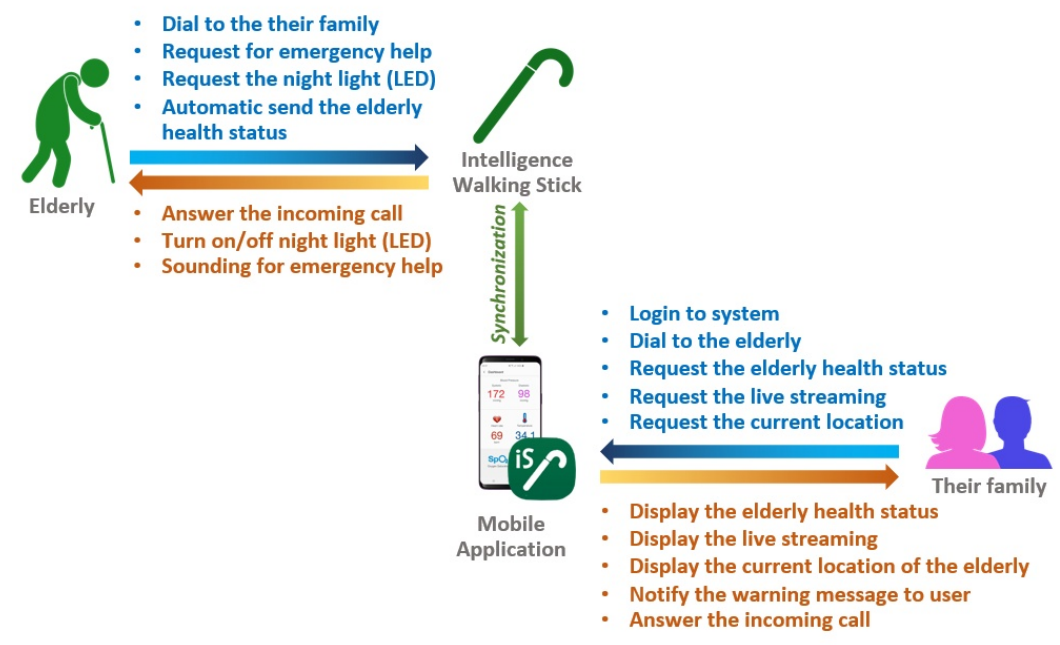

Fig. 3. The feature in the operation of the mobile application

\subsection{Development of the intelligence walking stick}

In this research, this system development can be separated into two parts. There are IoT development part and mobile application development part.

First, the IoT development, the researcher develops the application on the Arduino platform by using the Arduino IDE version 1.8.12 as editor and developer in the environment of the Windows 10 Professional Edition, with 64bits architecture. There are two main languages; $\mathrm{C}++$ and hypertext markup language (HTML). $\mathrm{C}++$ language is 
used to develop the main function for control all processes. The HTML is used to display the biological data from any sensors in this work. It developed for system initialization or re-configuration of the intelligence walking stick. By default, at the initialization stage, the station (STA) mode is default setup in this system. After that, all programming code uploaded into ESP32 board.

Second, mobile application development, it was developed on both iOS and Android operating system [13] which called cross-platform technology. A part of the mobile application was setting and initialize at the central server. This server acts as a data service provider that connects between a smartphone and an intelligent walking stick. The programming code based on Python version 3.6. There are five main functions of an intelligence walking stick: The programming code based on Python version 3.6. There are five main functions of an intelligence walking stick: 1) biological data measurement and display on the dashboard, 2) alert and alarm when the biological data is critical or exceed the threshold value, 3) real-time GPS tracking, 4) camera remote for live streaming or snapshot an image, and 5) calling or answering the emergency call from the elderly.

The mobile application screen display can illustrate in Figure 4 to Figure 8.

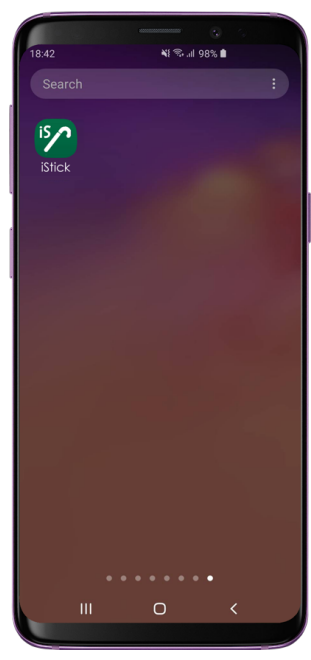

Fig. 4. The iStick application of an intelligence walking stick for the smartphone

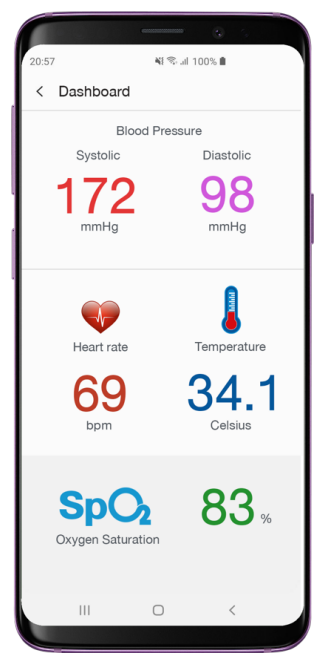

Fig. 5. The real-time biological data measurement 


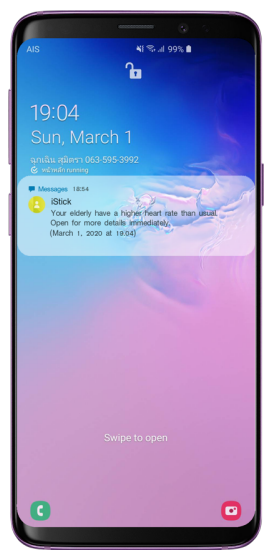

Fig. 6. The notification message when the biological data exceeds the threshold

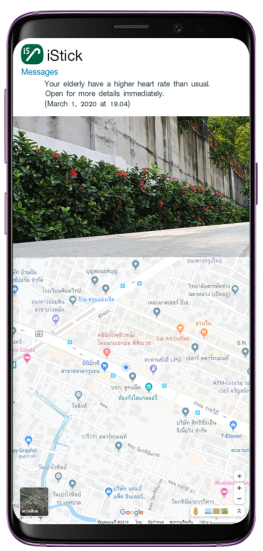

Fig. 7. GPS tracking the current location of the elderly

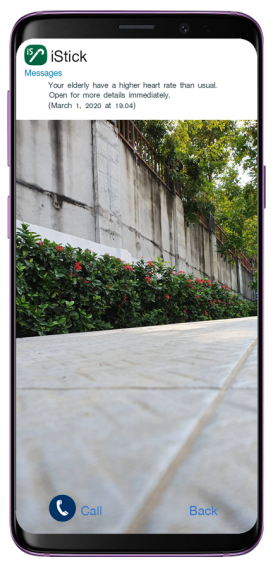

Fig. 8. The live streaming remotely by their family

\subsection{System usage training}

The training was provided to thirty-six people (eighteen elderly people and eighteen descendants) who were voluntarily participating in the mobile application test advertised on social networks. In this study, the participants received the documents explaining the protocols and ethical research conducts to sign. The 3-hours training involved a lecture, a demonstration, and real practice to try out the mobile application and intelligence walking stick. There was a guest speaker who was a lecturer in the field of information technology and nineteen information technology students as assistants. Both the guest speaker and the assistants had tested the developed mobile application. They had participated before in the academic service projects related to mobile applications and social media for the elderly community.

At the training begin, the guest speaker explained the online pre-test and advised participants via the projector. The training assistants conducted and guided each participant on how to access the online pre-test, which is formatted in the Google Form and is a multiple-choice of ten questions. However, the speakers and assistants did not encourage or direct the answers to the online pre-test to the participants. All participants had taken the online pre-test with content related to the features and function of the intelligence walking stick and the mobile application in half an hour. During the training process, the guest speaker was responsible for providing knowledge with PowerPoint slides through the projector and demonstrated how to use the intelligent walking stick and the mobile application. All participants have received documentation and instruction manuals that explain the installation process and how to use them 
thoroughly in conjunction with the training. The participants conducted practical exercises using the intelligent walking stick and the mobile application, while the guest speaker provides assistance and advice during training. After the training, each participant spent half an hour taking the online post-test, which had the same content as the online pre-test, but the difference is that there is an alternating sequence of questions and answer choices. In the final step of the training, when all participants have submitted all answers to the post-test, the guest speakers answered the answers, including the source of the training media, support manual, and links to download the mobile application. These links included the mobile application and intelligence walking stick demonstration video, which was published on social media for the elderly to watch and give the others information about the application and intelligence walking stick.

\subsection{The evaluation of the intelligence walking stick's effectiveness}

The effectiveness of the intelligence walking stick was evaluated by thirty-seven sample who were elderly people. Once the training ended, the people then evaluated the effectiveness of the intelligence walking stick in a survey on Google Form by focusing to applied the black box testing is used to evaluate the system of intelligence walking stick. In this research, there are five indicators evaluated by five experts whose expertise related to information technology or social science and fifteen elderly persons in Ratchaburi province. The four indicators for the black box testing are consist of: 1) functional testing, 2) compatibility testing, 3) usability testing and 4) security testing.

The data were analyzed to gain the mean value and the standard deviation (SD) value in regards to the Likert-scale scoring criteria [14], as illustrated in Table 2.

Table 2. Scoring criteria for evaluating the mobile application

\begin{tabular}{|c|c|l|}
\hline Scale & Range of Weighted Mean & \multicolumn{1}{|c|}{ Level of the effectiveness } \\
\hline 5 & $4.51-5.00$ & The highest \\
\hline 4 & $3.51-4.50$ & The high \\
\hline 3 & $2.51-3.50$ & The medium \\
\hline 2 & $1.51-2.50$ & The little \\
\hline 1 & $1.00-1.50$ & The least \\
\hline
\end{tabular}

\section{$3 \quad$ Results}

Five experts and thirty-seven sample users assessed the evaluation indicators of the developed intelligence walking stick. The data were analyzed to determine the mean and the standard deviation value based on the Likert-scale scoring criteria.

The effectiveness evaluation results of the intelligence walking stick that could be detected by experts while using the intelligence walking stick and mobile application showed that, among the five indicators, "functional testing" criteria had the highest mean value at 4.40 together with the standard deviation value at 0.55 , followed by 
"usability testing" with the mean value of 4.20 and the standard deviation of 0.84 . Next, "compatibility testing" and "security testing" received the mean value of 4.00 and the standard deviation value of 0.71 . The average mean value of 4.15 and the standard deviation value of 0.67 , showing that the developed intelligence walking stick was high effectiveness. Furthermore, the effectiveness evaluated by users, it showed that, among the five indicators, "functional testing" criteria had the highest mean value at 4.68 together with the standard deviation value at 0.47 , followed by "usability testing" with the mean value of 4.62 and the standard deviation of 0.49 . Next, "compatibility testing" received the mean value of 4.59 and the standard deviation value of 0.50 . Last, "security testing" received the mean value of 4.57 and the standard deviation value of 0.50 . The average mean value of 4.61 and the standard deviation value of 0.49 , showing that the developed intelligence walking stick was high effectiveness, as shown in Table 3.

According to Table 3, the result of evaluation for acceptance of the intelligence walking stick that could be detected by expert and elderly people while being used has high consensus. All of the values the interquartile range (IQR) no more than one and the quartile deviation (QD) no more than 0.5.

Table 3. The result of evaluation for acceptance of the intelligence walking stick that could be detected by experts and elderly people

\begin{tabular}{|c|c|c|c|c|c|c|c|}
\hline \multirow{2}{*}{ Assessment Indicators } & \multirow{2}{*}{ Mean } & \multirow{2}{*}{ SD } & \multicolumn{3}{|c|}{ Quartiles } & \multirow{2}{*}{ IQR } & \multirow{2}{*}{ QD } \\
\hline & & & $Q 1$ & Median & $Q 3$ & & \\
\hline \multicolumn{8}{|c|}{ Experts } \\
\hline 1. Functional testing & 4.40 & 0.55 & 4 & 4 & 5 & 0 & 0.5 \\
\hline 2. Compatibility testing & 4.00 & 0.71 & 4 & 4 & 4 & 0 & 0.0 \\
\hline 3. Usability testing & 4.20 & 0.84 & 4 & 4 & 5 & 1 & 0.5 \\
\hline 4. Security testing & 4.00 & 0.71 & 4 & 4 & 4 & 0 & 0.0 \\
\hline Total & 4.15 & 0.67 & 4 & 4 & 5 & 1 & 0.5 \\
\hline \multicolumn{8}{|c|}{ Users } \\
\hline 1. Functional testing & 4.68 & 0.47 & 4 & 5 & 5 & 1 & 0.5 \\
\hline 2. Compatibility testing & 4.59 & 0.50 & 4 & 5 & 5 & 1 & 0.5 \\
\hline 3. Usability testing & 4.62 & 0.49 & 4 & 5 & 5 & 1 & 0.5 \\
\hline 4. Security testing & 4.57 & 0.50 & 4 & 5 & 5 & 1 & 0.5 \\
\hline Total & 4.61 & 0.49 & 4 & 5 & 5 & 1 & 0.5 \\
\hline
\end{tabular}

\section{Conclusion}

This research purposes developing the intelligence walking stick for the elderly in terms of the health care system by applied the IoT devices and biometric sensors. This intelligence walking stick can detect and measure the biological data; there are heart rate, blood pressure, oxygen saturation, and the temperature in a real-time system. The MAX30101 sensor and MAX30208 sensor were used to detect and measure the biological data that mentioned above. All of the results of the measuring can monitor on the smartphone or mobile devices. This system was evaluated in the concept of black box testing in terms of functional, compatibility, usability, performance, and security 
testing. As a result of system evaluation, the intelligence walking stick system has the average mean value of 4.15 was high effectiveness with the standard deviation value of 0.67 , and the average mean value of 4.61 with the standard deviation value of 0.49 was highly effective by experts and users, respectively.

This study showed that technology could conveniently be applied to track the health of the elderly so they can provide emergency help in time through the intelligence walking stick and mobile applications to notify their family members. It is suitable for the current family situation in which elderly people tend to be alone. However, this study did reveal the vast possibilities of utilizing mobile technology and the internet of things to build better partnerships between the elderly and their family.

Future studies could include studies focusing on collecting and linking the health information of the elderly to public health in the village and the hospital where the elderly use regularly to facilitate doctors to monitor, follow up, diagnose the disease and provide help quickly.

\section{Acknowledgement}

We would like to express our gratitude to the Institute for Research and Development, Suan Sunandha Rajabhat University, who offered us the opportunities to conduct this research

\section{References}

[1] Ministry of Social Development and Human Security. (2010). Elderly Person Act, BE 2546. Bangkok: Theppenvanish Press.

[2] National Statistical Office. (2018). Report on the 2017 survey of the older persons in Thailand. Bangkok: National Statistical Office.

[3] OmronHealthcare. (2019). Omron blood pressure monitors. Retrieved February 22, 2020, from https://omronhealthcare.com/blood-pressure

[4] Samsung. (2018). Samsung Galaxy S9 specification. Retrieved February 5, 2020, from https://www.samsung.com/th/smartphones/galaxy-s9/specs https://doi.org/10.26439/ulima. tesis/8667

[5] OmronHealthcare. (2020). The premier wearable blood pressure monitor and much more. Retrieved February 19, 2020, from https://omronhealthcare.com/products/heartguide-wear able-blood-pressure-monitor-bp $8000 \mathrm{~m}$

[6] IdeaConnection. (2017, May 5). The iCane smart walking stick. Retrieved February 19, 2020, https://www.ideaconnection.com/new-inventions/the-icane-smart-walking-stick-118 $\underline{65 . h t m l}$

[7] Maxim Integrated. (2020). Max32664 ultra-low power biometric sensor hub. Retrieved March 9, 2020, https://www.maximintegrated.com/en/products/interface/sensor-interface/ MAX32664.html

[8] Rovinelli, R. J. and Hambleton, R. K. (1977). On the Use of Content Specialists in the Assessment of Criterion-Referenced Test Item Validity. Dutch Journal of Educational Research 
[9] Nuanmeesri, S. (2018). The augmented reality for teaching Thai students about the human heart. International Journal of Emerging Technologies in Learning. 13(6):203-213. https:// doi.org/10.3991/ijet.v13i06.8506

[10] Nuanmeesri, S. and Poomhiran, L. (2019). Perspective Electrical Circuit Simulation with Virtual Reality. International Journal of Online Engineering. 15(5):28-37. https://doi.org/ 10.3991/ijoe.v15i05.9653

[11] Nuanmeesri, S. (2019). Extended Study of Undergraduate Students' Usage of Mobile Application for Individual Differentiation Learning Support of Lecture-based General Education Subjects. International Journal of Interactive Mobile Technologies. 13(9):99-112. https://doi.org/10.3991/ijim.v13i09.10558

[12] Nuanmeesri, S. (2019). Mobile application for the purpose of marketing, product distribution and location-based logistics for elderly farmers. Applied Computing and Informatics. 9. https://doi.org/10.1016/j.aci.2019.11.001

[13] Shankah, G. (2020). The Devices of the Internet of Things Based on the Recognition of Handwriting Words with Mobile Assisted. International Journal of Interactive Mobile Technologies. 14(4):74-85. https://doi.org/10.3991/ijim.v14i04.12197

[14] Likert, R. (1932). A Technique for the measurement of attitudes. Archives of Psychology, New York University.

\section{$7 \quad$ Authors}

Sumitra Nuanmeesri is lecturer at Suan Sunandha Rajabhat University (SSRU), Bangkok 10130 Thailand. She has a Ph.D. in information technology from King Mongkut's University of Technology, North Bangkok. Her research interests include speech recognition, data mining, deep learning, image processing, web and mobile application, supply chain management system, augmented reality (AR) and virtual reality (VR) development, robotics, and the internet of things (IoT).

Lap Poomhiran is currently a Ph.D. student in Information Technology, Faculty of Information Technology, King Mongkut's University of Technology North Bangkok (KMUTNB), Bangkok 10800 Thailand. His research interests include web and mobile programming, augmented reality (AR) and virtual reality (VR) development, image processing, data mining, machine learning, deep learning, and the internet of things (IoT).

Article submitted 2020-04-13. Resubmitted 2020-05-28. Final acceptance 2020-05-29. Final version published as submitted by the authors. 